\title{
4 \\ Self-Harm as Violence: When Victim and Perpetrator Are One
}

Hanna Pickard

\section{Introduction}

Violence is standardly defined as behaviour involving physical force intended to hurt, damage or kill. There is no stipulation that the victim and perpetrator cannot be identical. Indeed, The World Report on Violence and Health is explicit that violence can be self-directed as well as other-directed (Krug et al. 2002). Based on this inclusion, it is estimated that 50\% of all deaths due to violence are self-inflicted, with $35 \%$ due to homicide and the remainder due to war or some other form of conflict (ibid.). Yet, to pick two illustrative examples, not one of the 41 chapters in The Cambridge Handbook of Violent Behaviour and Aggression addresses self-directed violence (Flannery et al. 2007), while the International Handbook of Violence Research devotes only one out of 62 chapters to suicide (Heitmeyer and Hagan 2003). Such collections aim to be far reaching and comprehensive compilations of state-of-the-art research into violence. Why does self-directed violence garner so little attention?

One natural answer to this question is that, despite the recognition by the World Health Organization (WHO) that violence can be self-directed, as a society we find it difficult to conceptualize self-harm and suicide as violence at all. One reason for this difficulty may be that our prototype of violence is other-directed. Think of violence, and the kinds of images that immediately spring to mind are likely to include fights, brawls, muggings, gang warfare, military warfare and perhaps sexual violence and the domestic abuse of women and children. But reflection on these images suggests a further reason for this difficulty, namely, that our prototype of the violent perpetrator is male.

Men are, indeed, perpetrators of more other-directed violence than women (Raine 2013). They are also almost twice as likely successfully 
to commit suicide than women (Krug et al. 2002). However, women are significantly more likely to attempt suicide than men (MHF 2007; Canetto and Sakinofsky 1998). The received explanation for this discrepancy appeals to the lethality of the means typically chosen by men, such as hanging, as opposed to women, such as overdosing (Canetto and Sakinofsky 1998; Schrijvers 2012). In addition, although there is some evidence that rates of male self-harm are rising (Kerr et al. 2010), women appear significantly more likely to self-harm than men (NICE 2011). It is estimated that five to six times as many adolescent girls self-harm as compared to boys (Hawton et al. 2012), and rates of self-harm in prisons in England and Wales are ten times higher for female than male inmates (Hawton et al. 2014). Moreover, just as our prototype of other-directed violence appears to be male, our prototype of self-harm appears to be female. Think, for instance, of Girl Interrupted, the film adaptation of Susanna Kaysen's memoir of the same name, or of Elizabeth Wurtzel's account of self-harm in her autobiography Prozac Nation.

Hence, we may find it difficult to conceptualize self-harm and suicide as violence, not only because it is self-directed but equally because, unlike other-directed violence, it is associated in our society with women. As feminist theorists since Simone de Beauvoir have documented with painstaking detail, within contemporary culture, women are regarded as 'the gentle sex' (de Beauvoir 1949 [1989]). Aggression and violence in men is typically viewed as natural. Aggression and violence in women, in contrast, is typically viewed as unnatural - a violation of our collective cultural archetype of the ideal woman as a loving, nurturing, mother figure (Motz 2008).

This image of women as naturally non-aggressive and non-violent arguably affects our understanding of violence. On the one hand, it may bar us from recognizing the very existence of female-perpetrated otherdirected violence, making us blind to incidences in which women are perpetrators. This tendency may be reinforced by the fact that most female other-directed violence occurs in the private realm, directed towards parents, partners and children, where it can more easily remain outside of public view (Motz 2008). On the other hand, when we recognize the existence of female-perpetrated violence of any form, the archetype of the ideal woman as naturally non-aggressive and non-violent may cause us to pathologize it. We may view violence in women, whether directed towards self or towards others, as an indication of perversion, deviance and sickness - an abomination of female nature.

Suicide and self-harm are indeed associated with mental health problems, and so easily pathologized (MHF 2007). Of course, both can and 
do occur outside of such contexts (Fincham et al. 2011). For instance, suicide may be a rational and dignified end-of-life choice for those who are terminally ill, or an act of political protest or moral or religious faith (Holroyd, this volume; Marway, this volume). Self-mutilation and the endurance of pain are a part of many religious, cultural and beautification rituals (Favazza 1987). However, in the context of mental health problems, an association between suicide and especially self-harm and women may have a particularly strong impact on our understanding of the nature of such behaviour. On the one hand, we may barely recognize it as violence at all. For our cultural assumption is that women are not violent. On the other hand, if we recognize it as violent, we may pathologize it as the very epitome of irrationality - as hysterical, emotional, incomprehensible behaviour. For, our cultural assumption is that, if women are violent, they are sick.

My aim in this chapter is to challenge this conception of suicide and especially self-harm, arguing that in central instances, it is on the one hand straightforwardly a form of violence, and, on the other, no more pathological than central instances of other-directed violence. Different people self-harm for different reasons, but given a realistic understanding of the psycho-socio-economic context which women who selfharm typically face, it emerges as fully comprehensible behaviour - an instrumental means to ends that, given the context, we can understand why women would have. In other words, self-harm emerges as a form of, broadly speaking, rational agency. As Anna Motz eloquently puts one component of this idea: 'To deny female violence is to deny female agency' (Motz 2008, 71). But to pathologize female violence is also to deny female humanity and rationality - to deny the way that aggression can be as natural for women to feel as it is for men, and that violence in women can function, as it does in men, not only to express aggression but also to serve various ends that embody clear and recognizable goods. This recognition is essential if we are to respond to self-directed violence in ways that are both effective and ethically appropriate.

The paper is structured as follows. In the next section, I begin by describing the nature of self-harm in order to establish, lest it be doubted, that it is indeed a form of violence. I then compare self-directed and otherdirected violence, sketching the differences and similarities. Drawing on epidemiological data, clinical understanding and first-person reports, I outline the various functions self-harm has, revealing its meaning through an understanding of the reasons why people do it. As already noted, the psycho-socio-economic context in which self-harm typically occurs is very important to this account. With this non-pathological 
understanding of self-harm as a form of violence in hand, I then turn in the final section to issues of agency, responsibility and ethics, sketching why and how we might hope to support and enable people to desist from self-harm, within clinical contexts and also through social and political agendas for change. I conclude by suggesting the need for a cultural shift, whereby we acknowledge that anger, aggression and the propensity for violence can exist in all of us, male and female, and so work together to fashion a culture that is able to better acknowledge and address this side of human nature.

\section{Self-harm as violence}

Self-harm, known also as self-injury, self-poisoning, self-mutilation and parasuicide, is not easy to define. The WHO defines it as 'an act with non-fatal outcome, in which an individual deliberately initiates a nonhabitual behaviour that, without intervention from others, will cause self-harm, or deliberately ingests a substance in excess of the prescribed or generally recognised dosage' (Kerkhof et al. 1994, 7). This definition aligns with common usage in its focus on the deliberate nature of self-harm, but is unusual in its claim that self-harm is a 'non-habitual behaviour' and will result in damage 'without intervention from others'. Self-harm is often a habit - a repetitive pattern of behaviour. Indeed, some people self-harm daily. Moreover, it is unclear why an absence of intervention by others is part of the definition at all - as if the possibility of self-harm depends fundamentally on such non-intervention, as opposed to depending fundamentally on the self-harmer's own agency.

In stark contrast to the WHO definition, the UK National Institute for Clinical Excellence defines self-harm as 'self-poisoning or self-injury, irrespective of the apparent purpose of the act' (NICE 2004, 2011). According to this definition, self-harm need not be deliberate. The motivation for this removal of intent is in all likelihood a desire to accommodate forms of self-harm that stem from unconscious or unrecognized motives or impulses, or that may be foreseeable but not directly intended. This definition therefore widens the scope of self-harm so that it can potentially include, for instance, anorexia and other eating disorders, substance abuse, sexual behaviour that carries health risks or makes one vulnerable to sexual abuse or violence, and even, arguably, actions like getting into fights or playing contact sports, where there is significant risk of physical damage even if that is not the apparent purpose of the activity. ${ }^{1}$ However, according to this definition, even genuine accidents that inadvertently result in self-injury but belie no unconscious motive 
and could not have been foreseen will count as self-harm. The WHO definition is too narrow, but the NICE definition is too wide.

Rather than focusing on defining self-harm, let us consider its central instances. Prototypical forms of self-harm include cutting, scratching or burning the skin; smashing one's body parts with weapons or banging them against walls; swallowing blades or sharp glass, or inserting them under the skin or in orifices; overdosing on illicit drugs, alcohol or prescribed medication, or ingesting poisons or toxic substances; tying ligatures around one's neck; or hanging oneself, shooting oneself or throwing one's body under trains, into oncoming traffic or off buildings. Some of these behaviours, of course, can also be forms of suicide. Suicide and self-harm can be distinguished in principle via intent: the intention in suicide is to die, while the intention in self-harm is not to die, but to damage or harm oneself. However, in reality the distinction between them is often unclear, with people unsure as to what they intend, or indifferent as to the outcome and reckless in method, so that self-harm can commonly and knowingly risk death, even if death is not the clear and conscious intention of the act. Indeed, self-harming behaviour is associated with a 50-100-fold increase in risk of suicide compared to the general population (NICE 2011).

Recall that violence is standardly defined as behaviour involving physical force intended to hurt, damage or kill. Although some of these prototypical forms of self-harm do not involve physical force, such as overdosing or ingesting poisons, most do. In central instances, self-harm is a form of behaviour directed at one's own body, which uses physical force to deliberately cause hurt and damage, if not death. Indeed, if we imagine the actions prototypical of self-harm to be other-directed, the violence inherent in them is palpable and shocking. As with the NICE definition, we can choose to extend the idea of self-harm to behaviour that is less direct in its means or less deliberate in its intention to cause hurt or damage, by analogy with these central instances, if that would serve a theoretical or clinical use. There are also potentially both theoretical and ethical reasons for widening the scope of violence, so that it both includes psychological damage as an outcome and also recognizes threats and exercises of power as nonphysical but yet violent means of perpetrating hurt or damage, by coercively securing victim compliance (Krug et al. 2002). But such caveats aside, central instances of self-harm show that it is prototypically direct and deliberate behaviour involving physical force inflicted by a person onto their own body with the intention of causing hurt or damage and even possibly risking death: it is violence where victim and perpetrator are one. 
Why would any person, man or woman, do this to themselves? We commonly assume that, if a person can act so as to avoid harm to themselves, they do. Arguably, this is a basic assumption of our culture's common sense or folk psychology - a reliable rule of thumb we use to explain and predict behaviour. But people who self-harm do precisely the opposite of this: they act to directly and deliberately harm themselves. Those of us who do not self-harm or have personal or professional experience with those who do may lack the resources to make sense of it. We may therefore find ourselves inclining to the view that the only way to understand self-harm is as an expression of pathology and irrationality. For, without an understanding of its functions, no sense can be made of why any person would do this if they could help it. This line of thought helps underwrite the conception of self-harm as hysterical, emotional, incomprehensible behaviour - as if the self-directed violence typical of women is a kind of sickness.

\section{Understanding self-harm in women}

To challenge this conception, it is useful to begin by comparing selfharm in women with our prototype of male-perpetrated other-directed violence. For it is not difficult for us to understand such other-directed violence as serving various functions. ${ }^{2}$ In addition to the use of violence as a direct means to procuring material goods, violence fulfils multiple individual and interpersonal psychological purposes. Most straightforwardly, especially male-on-male violence is often viewed as a natural masculine expression of an equally natural masculine aggression, stemming from emotions like anger or rage, arguably alongside fear of shame and vulnerability. Such male-on-male violence is often glorified in popular culture and the media, and may be part of the fabric of an individual's family and wider community, perhaps even necessary to survival and success within it. Even those of us who view violence as ethically unacceptable can understood the drive towards it, in the grip of aggressive impulses and strong emotions, within families and communities that endorse and expect it of men.

The ends served by other-directed violence include (but, of course, are not restricted to) the following:

(1) Affect regulation. The expression, release and communication of aggressive impulses and strong emotions, especially anger.

(2) Social and/or interpersonal dominance and/or control and the various benefits it accrues, especially in conformity with cultural stereotypes of masculinity. 
(3) Revenge. Retaliation/retribution towards those who have perpetrated psychological and/or physical harm to the agent or someone they care about.

(4) Protection from future harm. Violence can signal a willingness to retaliate/seek retribution and so act as a deterrent, when directed towards those who have perpetrated past harm or are threatening to perpetrate future harm.

Other-directed violence that flows from strong emotions, like anger, rage, shame and fear, is not typically the result of rational deliberation, understood as a conscious, reflective process of weighing the costs and benefits of various options, in order to decide what to do. But it is both typically rational in the sense that it is an instrumental means to these various ends, as well as subject to choice and a degree of control. ${ }^{3}$ The evidence for this is the classic 'policeman at the shoulder' test. Consider, for instance, a man who 'sees red' and routinely gets into conflicts and resorts to violence - except when in view of a policeman. On such occasions, he is highly motivated not to lash out, which he would otherwise do, lest he be detained and charged with common assault. The most natural understanding of this man's behaviour is that he has the capacity to choose not to be violent and control his aggression - a capacity, of course, that he only exercises when sufficiently motivated to do so. There is a basic, common-sense distinction between what a person can do but won't (because they don't want to) as opposed to what a person wants to do but can't (because they lack the capacity). In so far as other-directed violence is responsive to incentives, it appears to be subject to choice and a degree of control. Exercising this capacity and desisting from violence would therefore seem to be, in general, something people can do but sometimes don't, as opposed to something they want to do but can't. Very broadly, this is why we are typically inclined to view perpetrators of violence as rational agents whom it is appropriate to hold responsible and to account. ${ }^{4}$

However, it is extremely important to recognize how difficult it may be for people to exercise the capacity to desist from other-directed violence, especially when it is culturally expected if not indeed glorified, and in addition serves ends which, given the context and internal and external resources available, people may lack genuine or perceived alternative means of achieving. For example, insofar as other-directed violence offers affect regulation - a way of releasing, communicating and acting on strong emotions - refraining from it may require a person to undergo emotional distress and bear feelings of, for example, extreme anger, rage, anger, shame and fear, unless and until alternative ways of 
managing these strong emotions are learned. Men who have grown up in a cultural context which supports male aggression and violence as a way of managing emotions may have had little opportunity to learn other ways of dealing with these feelings. If so, although they retain choice and a degree of control when violent, we may yet feel these circumstances demand that we show them understanding and compassion, and offer help with emotional management, even if we are not prepared to excuse the violence. Equally, the general presumption that violence is subject to choice and a degree of control may, of course, be defeated in particular circumstances. Perhaps sometimes a person who 'sees red' becomes so angry that something 'boils over' or 'snaps' and choice and control is lost - they are not then able to stop the flow driving them towards violence. In such circumstances, if indeed a variety of complicated conditions are met, we may feel that the person ought to be (at least in part) excused, for they are not, in the moment, possessed of rational agency, but driven by forces they cannot control. ${ }^{5}$ But important as these caveats are, our basic cultural understanding of (especially male-on-male) other-directed violence is that it is expressive of rational agency: a means towards ends that are evidently desirable or valuable in our cultural context, and, although driven by strong emotions, yet subject to choice and a degree of control. No matter how much we may deplore it, we have ample cultural and folk psychological resources to make good sense of other-directed violence.

Although our cultural and folk psychological resources are less able to help us make sense of self-directed violence, it in fact serves remarkably similar ends to other-directed violence. Understanding its functions requires in the first instance contextualizing the behaviour, by appreciating the associated risk factors. These include (Hawton et al. 2012) the following:

(1) Sociodemographic and educational factors: female sex; low socioeconomic status; lesbian, gay, bisexual or transgender orientation; restricted educational achievement; exposure to self-directed violence through peer group.

(2) Psychiatric and psychological factors: mental disorder, especially personality disorder, depression, anxiety and ADHD; drug and alcohol misuse; impulsivity; low self-esteem; poor social problem solving; social isolation; perfectionism; hopelessness.

(3) Individual negative life events and family adversity: parental separation, divorce, discord, mental disorder or death; family history of suicide; interpersonal difficulties, especially childhood bullying; adverse childhood experiences, including a history of physical and, especially in women, sexual abuse. ${ }^{6}$ 
Women at risk of self-harm have typically come from underprivileged backgrounds of poor opportunity, and may have sexual orientations which make them vulnerable to ongoing social ostracization, discrimination, and violence. They are likely to have had difficult childhoods without good family or peer relationships and support, during which they may well have experienced emotional neglect, and physical and especially sexual victimization and abuse. As adults, they may struggle with mental health, and drug and alcohol problems, and have problems with relationships. Their educational and employment opportunities may be limited. And their self-esteem and self-worth are likely to be poor. In addition, like men who have been brought up in communities that fail to support nonviolent modes of male anger management, such women are also unlikely to have had the opportunity to learn positive ways of managing strong and difficult emotions and experiences during childhood, and may therefore lack both inner and outer resources for dealing effectively with them as adults.

Women who find themselves in this kind of psycho-socio-economic context will, of course, have very many good reasons for feeling anger and rage, both towards particular individuals and the world at large. They may also be subject to the shame and fear that is often associated with victimhood and sexual abuse in particular, and potentially to have internalized the negative attitudes towards them such mistreatment represents. Especially in a cultural context in which female aggression and violence are deemed unnatural, women cannot easily express or communicate their anger, rage or negative self-directed attitudes, or act publicly on their aggressive impulses, without violating gender expectations and norms. They may, therefore, turn these impulses inward onto their own bodies, enacting them in a private realm in which they can be kept hidden from public view.

It is important to emphasize that people self-harm for different reasons, but clinical understanding and patient self-reports reveal self-harm as serving at least six ends that, with this psycho-socio-economic background in mind, help explain why people engage in this behaviour. ${ }^{7}$

Self-directed ends of self-harm:

(1) Affect regulation. Like violence towards others, self-harm is a way of managing strong emotions, perhaps especially anger, rage and shame. It can offer relief in various ways: by distracting from emotional pain by replacing it with physical pain and/or releasing endorphins; by providing a way of expressing, releasing and acting on anger and aggression; or, in contrast, by allowing people to feel something in the face of dissociation and emotional numbing. In 
other words, self-harm can offer short-term relief from negative emotional experience of various forms: it is a coping mechanism.

(2) Self-punishment. Women who have internalized the negative attitudes towards them represented by a history of mistreatment typically have extremely low self-esteem and self-worth, and often at some level believe they are bad and deserve to be harmed. Self-harm can be both expressive of and explained by such negative self-directed attitudes.

Other-directed ends of self-harm:

(3) Communication. Self-harm can be a way of communicating internal distress by symbolizing emotions in concrete, physical form: 'the public expression of ... private pain' (Adshead 1997, 11). People who come from backgrounds of psycho-socio-economic adversity may struggle to identify and talk about their feelings. Self-harm offers a powerful way of demonstrating what they are going through, and, potentially, thereby seeking care and help.

(4) Other-punishment. It is natural, for women as much as for men, to feel anger and rage, and want to be aggressive and violent, towards those who have harmed either them or those whom they care about. Self-harm can offer a safe way of expressing such emotions and impulses, when violence towards others is deemed unacceptable. This function of self-harm correlates with the experience of being attacked that self-harm can provoke in others, as it becomes like a symbolic weapon, turning anger towards others inwards on the self, while yet communicating this anger to them.

Self-directed and other-directed ends of self-harm:

(5) Control. Self-harm can create a sense of empowerment and control by establishing ownership over one's own body in face of the experience of being helpless and violated. This function may be especially important for those with a history of childhood physical and sexual abuse. For people whose bodies have been harmed by others, it can be an act both of reclaiming their body and, to use Anna Freud's concept (1936 [1992]) of identification with the perpetrator, establishing that it is they, and no one else, who now has dominance and control.

A desire for death:

(6) The continuum with suicide. When associated with mental health problems, the desire to kill oneself is typically understood as 
expressive of hopelessness and despair - a desire for permanent escape from the suffering of living. In contrast, self-harm offers short-term relief, and hence, as Anna Motz has eloquently argued, can be seen instead as an act of hope (Motz 2009b) - an affirmation of life. However, as already noted in the previous section, although the idea of suicide is distinguished from self-harm by nature of intent - to die rather than to do harm or damage - in reality the distinction between them is often unclear. Self-harm regularly and knowingly risks death, due to indifference or recklessness, even if it does not clearly and consciously aim at it.

Self-harm is a response to psycho-socio-economic adversity and trauma, in a cultural context in which aggression and violence in women - what we might in men consider a natural response to such adversity and trauma - is deemed unnatural. Within this context, self-harm can function as a means to ends we can understand why women would have, such as feeling relief from anger, rage, shame and negative emotional experiences more generally, expressing and communicating, seeking care, attacking or punishing those perceived to have done wrong (including themselves, via internalization of the negative attitudes expressed through their past mistreatment), and gaining a sense of power and control in the face of feelings of helplessness and violation.

Like other-directed violence that flows from strong emotions, selfdirected violence is not typically the result of rational deliberation. But it is typically both rational in the sense that it is an instrumental means to these various ends, as well as subject to choice and a degree of control. The evidence for this, again as with other-directed violence, is that, despite the extreme difficulty in desisting from self-directed violence, especially in the absence of genuine or perceived alternative means of achieving the various ends it fulfils, women who self-harm may choose not to do so when self-harm would conflict with other ends they value more. To take a common example, the classic 'policeman at the shoulder test' can here be transposed to a 'child at the shoulder test' when, for instance, women who self-harm ensure that their children never witness their self-directed violence, out of care and concern for the impact it would have on them. Another kind of example regularly occurs when women stop 'cold turkey' as a condition of joining a therapy group, much along the model of support groups for addiction, such as Alcoholics Anonymous. ${ }^{8}$ Hence self-directed violence in women, like other-directed violence in men, is not an expression of pathology but an expression, at least in good part, of rational agency: a means to ends 
we can understand why women who have suffered adversity, trauma and abuse would have, and subject to choice and a degree of control. Consider, in this light, the following first-person report:

I needed to kill something in me, this awful feeling like worms tunneling along my nerves. So when I discovered the razor blade, cutting, if you'll believe me, was my gesture of hope. All the chaos, the sound and fury, the confusion and uncertainty and despair - all of it evaporated in an instant and I was for that minute grounded, coherent, whole. Here is the irreducible self. I drew the line in the sand, marked my body as mine, its flesh and its blood under my command. ${ }^{9}$

It is a harrowing indictment of our society that women suffer such adversity, trauma and abuse, and are possessed of so few genuine or perceived alternative options, that they choose to be violent towards themselves as a way of coping with and expressing their experience. Unlike violence towards others, we do not typically view self-directed violence as morally wrong, nor, since the legalization of suicide, is it criminalized. ${ }^{10}$ But it does violate a basic tenet of any broadly liberal society, which is that respect and equality are ideally due to all persons, irrespective of who they are. ${ }^{11}$ The very universality of this tenet means that, from each of our perspectives, it must apply not only to others but also to ourselves. Women who self-harm seem not to treat themselves with the respect and equality that, given this ethical outlook, they along with all other persons are due. Situating self-harm within its psycho-socio-economic context can help explain why women treat themselves and their bodies with such disregard and brutality: part of the explanation is that this may be how they and their bodies have been treated by others. Effectively and ethically addressing self-harm therefore has two interwoven facets. One facet looks to the rational agency of the self-harmer herself, and the need to support and empower her to choose self-care over self-harm. The other facet looks to the context of suffering, adversity and oppression in which women are situated, and the need to change the ways they are treated in wider society and the options available to them.

\section{Agency, ethics, and the validation and tolerance of anger}

Alongside the tendency to pathologize self-harm and deny the selfharmer's rational agency is a corresponding tendency to treat her only as a victim, helpless and out of control, possibly rendered hysterical by her 
emotions. But women who self-harm are rational agents of self-directed violence - they are not only victims. Effective clinical interventions and other forms of interpersonal engagement aimed at self-harm cessation must acknowledge that women who self-harm do so for reasons, and have choice. For they need to exercise their control and choose to do things differently in order to stop - by deciding to resist the desire to self-harm, find alternative ways to fulfil the ends it serves, and, in addition, question and challenge some of the more self-denigrating ends self-harm may serve and express. When the desire to self-harm is present and the behaviour habitual, cessation almost invariably requires the exercise of rational agency: broadly speaking, women must choose selfcare over self-harm.

But clinical interventions that acknowledge a person's rational agency inevitably carry a risk. In this case, the risk is that in acknowledging and working with women as agents of self-harm, clinicians may end up judging and blaming the self-harmer for her actions, and experiencing or expressing a range of critical, negative attitudes and emotions. Despite the fact that self-harm is neither criminalized nor intuitively viewed as morally wrong, it is, after all, a form of violence, and it can have a profound impact on other people, especially when it is perceived as an expression of anger and rage, or a symbolic attack. Rational agency carries the (defeasible) assignment of responsibility, and it can be an easy slide from an acknowledgement of agency and (potentially) responsibility, to blaming attitudes and emotions, such as disapproval, dislike, rejection, contempt, disgust and anger - as if 'the problem' lies fundamentally with the self-harmer herself.

To be effective, clinical engagement must acknowledge agency and (potentially) responsibility, while at the same time avoiding blame and maintaining care, concern and respect for women who self-harm. A blaming atmosphere is likely to re-enact and reinforce past mistreatment and its impact on women, destroying the possibility of building trust and understanding. In contrast, a stable environment that maintains care, concern and respect potentially offers women a corrective experience, ideally helping them overcome the impact of past mistreatment on their self-esteem and self-worth, through therapeutic relationships that validate them and their experiences, and consistently and authentically show compassion and positive regard. In such environments, women may feel supported and empowered to stop self-harming, and embark on a process of freeing themselves from past mistreatment and learning new ways of managing feelings and caring for themselves. ${ }^{12}$ Given the extent of past mistreatment, this may be a long and painful 
process, requiring women to have the courage to face traumatic aspects of their past in order to develop a narrative understanding of its impact on their present, thereby enabling them to identify patterns and make changes to improve their lives. ${ }^{13}$

There are many features of the clinical environment that help avoid any inclination to blame that may arise, and to maintain care, concern and respect. In this regard, it is undoubtedly crucial that the basic clinical aim is to help patients, and that this aim structures clinical practices, roles and relationships, shaping a culture of care. But this culture is also aided by an awareness of the psycho-socio-economic past and present context of women who self-harm. Attention to this context is necessary in any case to the development of a narrative understanding of the person and to the facilitation of realistic change. But it also acts as an antidote to any inclination to blame, undercutting it directly by situating self-harm in relation to underprivileged psych-socio-economic contexts, and thereby forcing it to exist alongside the attitudes and emotions that recognition of such inequality and injustice engenders. For 'the problem' does not lie fundamentally with the self-harmer herself, but with the context of suffering, adversity and oppression in which women who self-harm are typically situated. ${ }^{14}$

Alongside supporting and empowering women to choose self-care over self-harm, effectively and ethically addressing self-harm therefore requires challenging psycho-socio-economic contexts that harm women. The basic tenet of any broadly liberal society, that respect and equality are ideally due to all persons irrespective of who they are, supports this wider social and political agenda for change for people of all genders: we must address suffering, adversity and oppression. Such an agenda for change may be preventive with respect to self-harm on a large scale: associations are not the same as causes, but nonetheless, we should expect that addressing some of the known risk factors may have an impact on rates of self-harm. But it is also important for enabling individual women who self-harm to successfully stop: clinical interventions are significantly less likely to be effective if women leave clinical contexts only to return to psycho-socio-economic contexts in which they are again subjected to inequality and injustice, and which therefore re-enact and reinforce precisely the mind-set and habits that clinical interventions aim to change.

Hence, effectively and ethically addressing self-harm, as well arguably as other-directed violence, requires working with individuals alongside working to change wider social and political systems. But I want to conclude by briefly commenting on how, at a broad cultural level, what 
needs to change may include our cultural attitudes towards anger with respect to both men and women: it may not only be inequality and injustice that need to be addressed, but also our tendency to condemn and suppress people's emotions.

In contemporary culture, anger is viewed as natural in men, but so too is aggression and violence - indeed, there may be few non-aggressive and non-violent socially acceptable models of behaviour available to men to deal with angry feelings. For women, in contrast, anger itself - never mind the aggression and violence it can prompt - may be treated as unnatural and indeed unacceptable: anger may be thought 'unbecoming' in 'the gentle sex'. An alternative and opposing cultural outlook maintains that anger - no doubt alongside other difficult emotions, such as shame and fear - and the aggression and violence it drives is natural for all people of all genders, especially when we find ourselves in contexts where we have been harmed. If that harm is perpetrated by others through mistreatment, neglect of our needs, outright violation and abuse, or other forms of disrespect, subjugation or inequality, we may feel angry and want to be aggressive or violent towards the perpetrators. If the harm is more a product of bad luck or wider social and political circumstances, lacking an identifiable and responsible agent, we may yet feel angry and want to be aggressive and violent in a more inchoate or undirected way, and understandably so. Either way, anger and the propensity for aggression and violence are natural human responses to harm and wrongdoing - found in people of all genders equally.

But in addition to differential treatment of men and women, contemporary culture seems also to fail in general to draw a clear distinction, with respect to all people, between anger and other difficult emotions on the one hand, and the behaviour that these emotions can drive on the other. In men, anger, aggression and violence may be accepted, but all equally condemned as wrong. In women, anger, aggression and violence are not accepted but culturally suppressed, as well as condemned as wrong. But an alternative and opposing cultural outlook maintains that, to the contrary, with respect people of all genders, there is nothing wrong with feeling angry or indeed feeling any other difficult emotion even if it is indeed often wrong to act aggressively or violently.

This alternative outlook is in fact realized in clinical and other contexts that are designed to heal wounds or resolve conflicts: the need to acknowledge, validate and tolerate anger and other difficult emotions is recognized as crucial, in order to enable people who have been harmed to feel respected, cared for, and that their experience of suffering or 
mistreatment is recognized and taken seriously by others. ${ }^{15}$ So too, as part of a process of validation and tolerance, is providing people with authentic means of expression, communication and action in relation to such feelings, as alternatives to aggression and violence. ${ }^{16}$ Contemporary culture notwithstanding, the existence of such contexts and practices establishes that it is possible for people to speak their anger and show how they feel, without resorting to aggression and violence. Especially when situated within psycho-socio-economic contexts of suffering and oppression, anger is understandable and legitimate. Perhaps if anger and other difficult emotions were better validated and tolerated in our wider culture, with respect to people of all genders, it would be more possible for all of us to find means of expressing, communicating and acting on our feelings, such that we resorted to violence - whether other-directed and self-directed - less. ${ }^{17}$

\section{Notes}

1. It is both interesting and potentially clinically useful to consider whether these latter sorts of behaviours represent ways in which men are able to self-harm in our culture, under the more socially accepted masculine guise of otherdirected aggression and violence. Fight Club, the film adaptation of Chuck Palahniuk's novel of the same name, arguably presents male fighting in this light.

2. It is important to distinguish what has been labelled 'reactive' or 'hot' violence, from what has been labelled 'instrumental' or 'cold and calculating' violence and which is associated with psychopathy. For discussion of this distinction and the empirical data supporting it, see e.g. Blair et al. 2005, Howell 2009, and Raine 2013. My focus in this article is on 'reactive' or 'hot' violence as opposed to psychopathic violence. However, the labelling is unfortunate, as reactive violence is also instrumental, in the sense of serving various functions or ends, as discussed above.

3. For an important discussion of the difference between rational deliberation and choice, see Holton 2006. Pickard 2015 offers a more detailed discussion of deliberation and choice in self-directed and other-directed violence committed by people with personality disorder, in relation to both mental health and criminal law.

4. For further discussion of this form of argument, especially in relation to the nature of addiction on the one hand, and personality disorder, and criminal law on the other, see Pickard 2012, 2013a, 2013b, and 2015.

5. Holton and Schute 2007 draw on philosophy and psychology to understand the nature of loss of control and link it to the older defence of provocation in English law. Although this defence was abolished and replaced with a loss of self-control defence under The Coroners and Justice Act 2009 Sections 54-6, which widens the emotions relevant to the defence, Holton and Schute's discussion of the philosophy and psychology is yet relevant. See too Horder 2005 for discussion of the role of fear alongside anger in loss of self-control. 
6. Note that a history of childhood physical abuse is associated with otherdirected violence in men, while a history of childhood sexual abuse is associated with self-directed violence in women (Waxman et al. 2013).

7. See Motz 2009a for an excellent recent collection of articles about self-harm, particularly in relation to forensic contexts. See too Motz 2008, especially chapter 6, and the NICE Guidelines 2011 for longer-term management of self-harm. There are also an increasing number of patient self-reports available online and in medical textbooks and guidelines. Hawton et al. 2012 is a comprehensive review of medical and social science studies of self-harm in adolescents. See Klonsky 2007 for a review of the evidence outlining the functions of self-harm.

8. For discussion, see Pickard 2013a and 2013b.

9. First-person report, quoted in Motz 2009a, 47.

10. Interestingly, the claim that self-harm is not morally wrong can be found within the philosophical literature on ethics. Cf. Pritchard: 'It may be noted that if the badness of pain were the reason why we ought not to inflict pain on another, it would equally be a reason why we ought not to inflict pain on ourselves; and yet, though we should allow the wanton infliction of pain on ourselves to be foolish, we should not think of describing it as wrong' (1912 [1949], 5). Cf. too Slote: 'ordinary moral thinking seems to involve an asymmetry regarding what an agent is permitted to do to himself and what he is permitted to do to others. If one can easily prevent someone else's pain, it is typically thought wrong not to do so, but not to avoid similar pain in oneself (... or indeed, to inflict it we might add ...) is only lazy, crazy, or senseless, not wrong' $(1984,181)$. Note that, in addition to denying the immorality of self-harm, both these philosophers also characterize it - in keeping with its conception as pathological or sick - as foolish, crazy, and senseless.

11. For the classic statement of this liberal position, see Dworkin 1977 and 1985.

12. For a classic discussion of the principles of working effectively with selfharm, see Tantum and Whittaker 1993. Lawday 2009 and Grocutt 2009 are excellent discussions of self-harm cessation in secure settings, applicable to non-secure settings too. The NICE Guidelines 2004 and 2011 as well as Wood et al. 2014 also offer invaluable resources.

13. For discussion of the role of narrative in effective therapeutic processes, see Pickard 2014.

14. For further discussion of the distinction between responsibility and blame, in theory and in practice, alongside other themes of this section see Pickard 2011 and 2013b, and Lacey and Pickard 2013. The importance of psychosocio-economic context is also emphasized by feminist theories of relational autonomy, which argue that in such conditions, autonomy is impaired. Stoljar 2014 offers an excellent survey of the extensive literature; see Benson 1994 and Govier 1993 for discussions particularly relevant to issues arising in relation to self-harm, because of their focus on how oppression impacts on selfworth and self-trust, understood as a precondition of autonomy. However, to grant that such conditions impair autonomy does not change the fact that, from a pragmatic perspective, effective means of addressing self-harm need to work with the self-harmer's own rational agency as well as acknowledging and addressing psycho-socio-economic context. For irrespective of the impact of these conditions on autonomy, they do not nullify rational agency, which 
is therefore a powerful and essential resource for women who self-harm, as it is for others who are subject to oppression of various forms.

15. Important non-clinical examples arguably include some forms of Restorative Justice Congress as well as the South African Truth and Reconciliation Commission. For discussion of the role of emotions in restorative justice practices, see Rossner 2013.

16. The non-violent communication skills developed by Marshall Rosenberg and used for conflict resolution, mediation and peacekeeping constitute an internationally recognized example of such a practice. Rosenberg has written many books describing the techniques and benefits of non-violent communication, but for an introduction which relates it in particular to the validation and toleration of anger, see his 2005.

17. I am grateful to Anna Motz, Ian Phillips and Amia Srinivasan for discussion of the themes of this paper. This research is funded by the Wellcome Trust [grant number 090768].

\section{References}

Beauvoir de, S. 1949 [1989]. The Second Sex. New York: Knopf.

Benson, P. 1994. 'Free agency and self-worth', Journal of Philosophy, 91, 650-668.

Blair, J., Mitchell, D. and Blair, K. 2005. The Psychopath: Emotion and the Brain. Malden, MA: Blackwell.

Canetto, S.S. and Sakinofsky, I. 1998. 'The gender paradox in suicide', Suicide and Life-Threatening Behaviour, 28, 1-23.

Dworkin, R. 1977. Taking Rights Seriously. Cambridge, MA: Harvard University Press.

Dworkin, R. 1985. A Matter of Principle. Cambridge, MA: Harvard University Press.

Favazza, A. 1987. Bodies under Siege. Baltimore: John Hopkins University Press.

Fincham, B., Langer, S., Scourfield, J., and Shiner, M. 2011. Understanding Suicide: A Sociological Autopsy. New York: Palgrave Macmillan.

Flannery, D.J., Vazsonyi, A.T., and Waldman, I.D. 2007. The Cambridge Handbook of Violent Behaviour and Aggression. New York: Cambridge University Press.

Freud, A. 1936 [1992]. The Ego and the Mechanisms of Defence. London: Karnac Books.

Govier, T. 1993. 'Self-trust, autonomy, and self-esteem', Hypatia, 8, 99-120.

Grocutt, E. 2009. 'Self-harm Cessation in Secure Settings' in A. Motz (ed.) Managing Self-harm: Psychological Perspectives. Hove: Routledge, 180-203.

Hawton, K., Linsell, L., Adeniji, T., Sariaslan, A., and Fazel, S. 2014. 'Self-harm in prisons in England and Wales: an epidemiological study of prevalence, risk factors, clustering and subsequent suicide', The Lancet, 383, 1147-1154.

Hawton, K., Saunders, K.E.A., and O'Connor, R.C. 2012. 'Self-harm and suicide in adolescents', The Lancet, 379, 2373-2382.

Heitmeyer, W., and Hagan, J. 2003. The International Handbook of Violence Research. The Netherlands: Kluwer Academic Publishers.

Holton, R. 2006. 'The act of choice', Philosophers' Imprint, 6, 1-15.

Holton, R. and Shute, S. 2007. 'Self-control in the modern provocation defence', Oxford Journal of Legal Studies, 27, 49-74. 
Horder, J. 2005. 'Reshaping the subjective element in the provocation defence', Oxford Journal of Legal Studies, 25, 123-140.

Howells, K. 2009. 'Angry Affect, Aggression, and Personality Disorder' in M. McMurran and R.C. Howard (eds.) Personality, Personality Disorder, and Violence. Chichester: John Wiley \& Sons, 191-212.

Kerkhof, A.J.F.M, Schmidtke, A., and Bille-Brahe, U. 1994. Attempted Suicide in Europe: Findings from the Multicentre Study on Parasuicide by the WHO Regional Office for Europe. Leiden: DSWO Press.

Kerr, P.L., Muehlenkamp, J.J., and Turner, J.M. 2010. 'Nonsuicidal self-injury: A review of current research for family medicine and primary care physicians', American Board of Family Medicine, 23, 240-259.

Klonsky, E.D. 2007. 'The functions of deliberate self-injury: a review of the evidence', Clinical Psychological Review, 27, 226-239.

Krug, E.G., Dahlberg, L.L., Mercy, J.A., Zwi, A.B, and Lozano, R. 2002. World Report on Violence and Health. Geneva: World Health Organization.

Lacey, N., and Pickard, H. 2013. 'From the consulting room to the court room? Taking the clinical model of responsibility without blame into the legal realm', Oxford Journal of Legal Studies, 33, 1-29.

Lawday, R. 2009. 'Self-Harm in Women's Secure Services: Reflections and Strategies for Treatment Design' in A. Motz (ed.) Managing Self-harm: Psychological Perspectives. Hove: Routledge, 157-179.

Mental Health Foundation (MHF). 2007. The Fundamental Facts. London, UK: MHF.

Motz, A. 2008. The Psychology of Female Violence: Crimes against the Body. Hove: Routledge.

Motz, A. (ed.) 2009a. Managing Self-harm: Psychological Perspectives. Hove: Routledge.

Motz, A. 2009b. 'Self-harm as a Sign of Hope', in A. Motz (ed.) Managing Self-harm: Psychological Perspectives. Hove: Routledge, 15-41.

National Institute for Clinical Excellence. 2004. Self-Harm: The Short-Term Physical and Psychological Management and Secondary Prevention of Self-Harm in Primary and Secondary Care (CG 16). London: NICE.

National Institute for Clinical Excellence. 2011. Self-Harm:Longer-Term Management (CG 133). London: NICE.

Pickard, H. 2011. 'Responsibility without blame: empathy and the effective treatment of personality disorder', Philosophy, Psychiatry, Psychology, 18, 209-233.

Pickard, H. 2012. 'The purpose in chronic addiction', American Journal of Bioethics Neuroscience, 3, 40-49.

Pickard, H. 2013a. 'Psychopathology and the ability to do otherwise', Philosophy and Phenomenological Research. First published online 8 April 2013. DOI:10.1111/ phpr.12025.

Pickard, H. 2013b. 'Responsibility without Blame: Philosophical Reflections on Clinical Practice' in K.W.M. Fulford, M. Davies, R.T. Gipps, G. Graham, J. Sadler, G. Strangellini, and T. Thornton (eds) The Oxford Handbook of Philosophy of Psychiatry. Oxford: Oxford University Press, 1134-1152.

Pickard, H. 2014. 'Stories of Recovery: The Role of Narrative and Hope in Overcoming PTSD and PD' in J.Z Sadler, K.W.M. Fulford, C.W. van Staden (eds) The Oxford Handbook of Psychiatric Ethics. Oxford: Oxford University Press. Online Publication Date: October 2014. DOI: 10.1093/ oxfordhb/9780198732365.013.30. 
Pickard, H. 2015. 'Choice, deliberation, violence: mental capacity and criminal responsibility in personality disorder', International Journal of Law and Psychiatry, 40, 15-24.

Pritchard, H.A. 1912 [1949]. 'Does moral philosophy rest on a mistake?' In Moral Obligation: Essays and Lectures. Oxford: Clarendon Press, 1-17.

Raine, A. 2013. The Anatomy of Violence: The Biological Roots of Crime. London: Penguin.

Rosenberg, M. 2005. The Surprising Purpose of Anger - Beyond Anger Management: Finding the Gift. Encinitas CA: PuddleDancer Press.

Rossner, M. 2013. Just Emotions: Rituals of Restorative Justice. Oxford: Oxford University Press.

Schrijvers, D. 2012. 'The gender paradox in suicidal behavior and its impact on the suicidal process', Journal of Affective Disorders, 138, 19-26.

Slote, M. 1984. 'Morality and self-other asymmetry', Journal of Philosophy, 81, 179-192.

Stoljar, N. 2014. Feminist Perspectives on Autonomy. The Stanford Encyclopedia of Philosophy (Spring 2014 Edition). E.N. Zalta (ed.) Available at: http://plato. stanford.edu/archives/spr2014/entries/feminism-autonomy/.

Tantum, D., and Whittaker, J. 1993. 'Self-Wounding and Personality Disorder' in P. Tyrer and G. Stein (eds.) Personality Disorder Reviewed. London: Gaskell, 191-224.

Waxman, R., Fenton, M.C., Skodol, A.E., Grant, B.F., and Hasin, D. 2014. 'Childhood maltreatment and personality disorders in the USA: Specificity of effects and the impact of gender', Personality and Mental Health, 8, 30-41.

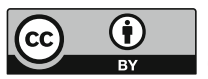

Except where otherwise noted, this chapter is licensed under a Creative Commons Attribution 3.0 Unported License. To view a copy of this license, visit http://creativecommons.org/licenses/by/3.0/ 